\title{
Mid-term results of deep vein thrombosis treatment: Comparison of interventional and medical therapies
}

\author{
Zeynep Gülben Kük ${ }^{1}$, Kamil Boyacıoğlu² (D, Bülent Mert² ${ }^{2}$, Adil Polat ${ }^{2}$ (D) \\ ${ }^{1}$ Department of Cardiovascular Surgery, Tatvan State Hospital, Bitlis, Turkey \\ ${ }^{2}$ Department of Cardiovascular Surgery, University of Health Sciences, Bağcllar Training and Research Hospital, Istanbul, Turkey
}

\begin{abstract}
Objectives: This study aims to compare different modalities used in the treatment of deep vein thrombosis (DVT) and to identify risk factors for post-thrombotic syndrome (PTS).

Patients and methods: A total of 217 patients (119 males, 98 females; mean age $51.2 \pm 18.1$ years; range, 15 to 96 years) who were treated for DVT with medical and interventional methods between August 2012 and September 2018 were retrospectively analyzed. The interventional group (Group I) consisted of 35 patients and the medical group (Group M) consisted of 182 patients. The patients were compared for risk factors, anatomic extension, and final clinical pictures.

Results: The mean follow-up was $2.6 \pm 2.5$ years. Group I had more proximal involvement according to the Lower Extremity Thrombosis (LET) classification ( $p=0.0001)$. Recurrent DVT rates were 22.9\% $(n=8)$ and $13.9 \%(n=23)$ in Group I and Group M, respectively ( $p=0.198)$. The mean recurrence-free survival duration in Group I and Group M was 1.6 \pm 1.7 and 2.6 \pm 3.7 years, respectively ( $\mathrm{p}=0.052$ ). Two-year recurrence-free survival rates in Group I and Group $M$ were $73.3 \pm 9.8 \%$ and $85.9 \pm 3.7 \%$, respectively ( $p=0.479$ ). None of the Group I patients had pulmonary embolism (PE) during follow-up. In Group M, five patients had PE ( $\mathrm{p}=0.614)$. In the final controls, the mean Villalta scores in Group I and Group M were 3.0 \pm 2.6 and 5.4 \pm 5.2 , respectively ( $\mathrm{p}=0.013$ ). The rates of patients with Villalta scores $>5$ were $3.4 \%$ ( $\mathrm{n}=1$ ) in Group I and 41.0\% ( $\mathrm{n}=32)$ in Group M ( $\mathrm{p}=0.0001)$. The mean Venous Clinical Severity Scores (VCSS) were statistically significantly different in Group I and Group M (2.6 \pm 3.5 and 4.4 $\pm 3.6 ; p=0.027)$. Anticoagulant-related bleeding complication rates in Group I and Group M were $27.6 \%(n=8)$ and $41.0 \%(n=32)(p=0.195)$.
\end{abstract}

Conclusion: Interventional DVT treatment results in decreased symptom severity and PTS occurrence at two years. Interventional therapy options in iliofemoral DVT offers persistent advantage in the mid-term.

Keywords: Deep vein thrombosis, post-thrombotic syndrome, transcatheter thrombectomy.

Deep vein thrombosis (DVT) is a disease with $0.05 \%$ incidence. ${ }^{[1]}$ Immobility, trauma, elderly age, surgical operation, and malignancy are predisposing factors for DVT. Deep vein thrombosis is an important cause of mortality and morbidity associated with pulmonary embolism (PE) and post-thrombotic syndrome (PTS). Anticoagulation is the mainstay of DVT treatment. Most patients with DVT can be treated in the outpatient setting with low-molecularweight heparin (LMWH) and vitamin $\mathrm{K}$ antagonist (VKA) or direct-acting oral anticoagulants (DOACs). Catheter-directed thrombolysis (CDT) is an alternative option for the treatment DVT and is known to decrease the incidence of PTS. Patients who are most likely to benefit from CDT have iliofemoral DVT, symptoms for $<14$ days, good functional status, a life expectancy of $>1$ year, and a low risk for bleeding. ${ }^{[2]}$

The acute venous thrombosis: Thrombus Removal with Adjunctive Catheter-Directed Thrombolysis (ATTRACT) trial was designed to test whether interventional treatments had advantages over medical treatment. ${ }^{[3]}$ In this study, the follow-up results of patients undergoing interventional and medical treatment options at 24 months were analyzed.

Received: June 11, 2019 Accepted: August 01, 2019 Published online: September 16, 2019

Correspondence: Adil Polat, MD. SBÜ Bağcllar Ĕ̆itim ve Araştırma Hastanesi Kalp ve Damar Cerrahisi Kliniği, 34100 Bağcllar, İstanbul, Turkey. e-mail: dradilpolat@gmail.com 
All patients with iliofemoral and femoropopliteal thrombosis were included in the study, and no significant difference was found in terms of the development of PTS and recurrent DVT. The patients with iliofemoral involvement had lower rates of PTS (25\%) and bleeding rates were found to be lower and no mortality was observed. ${ }^{[3]}$

Our mid-term results based on ultrasoundaccelerated CDT experiences have been previously published. ${ }^{[4]}$ In the present study, we aimed to compare medical and interventional modalities used in the treatment of DVT and to identify risk factors for PTS.

\section{PATIENTS AND METHODS}

This retrospective study included a total of 217 patients (119 males, 98 females; mean age $51.2 \pm 18.1$ years; range, 15 to 96 years) with DVT who received medical and interventional methods between August 2012 and September 2018. Data were obtained from the hospital records and telephone calls. The final functional status and clinical presentation of the patients were obtained through telephone interviews with outpatient examination records. In all patients, malignancy, immobility, traumas, history of orthopedic surgery, other major surgical interventions, previous vascular surgery, pregnancy and contraception, coagulopathies, long journey, oral contraceptive use, advanced age, obesity, rheumatologic diseases, working conditions were questioned. Deep vein thrombosis was considered provoked in the presence of a risk factor, while it was considered non-provoked, if no risk factor was present. The patients who had $>5$ points of Villalta scores, 8 points of Venous Clinical Severity Score (VCSS) or who had venous ulcer were accepted as having PTS. In the presence of positive medical history for $\mathrm{PE}$, the patients were considered to have PE. The predisposing factor was accepted to be present within two months before the development of DVT in trauma cases. Malignancies were not considered as a predisposing factor, if the cure was six months or longer. Pregnancy and postpartum (the first two months after delivery) periods were considered predisposing factors for DVT. We classified the levels of involvement in relation to the first ultrasound imaging according to the Lower Extremity Thrombosis (LET) Classification. ${ }^{[5]}$

The patients were divided into two groups: the interventional group (Group I, $\mathrm{n}=35$ ) receiving interventional methods such as surgical, mechanical, or pharmacomechanical thrombectomy and the medical group (Group $M, n=182$ ) receiving medical treatment alone. A written informed consent was obtained from each patient. The study protocol was approved by the Ethics Committee of Clinical Researches of Health Sciences University, Faculty of Medicine, Bağcrlar Training and Research Hospital (Protocol No: 2018.08.2.03.080). The study was conducted in accordance with the principles of the Declaration of Helsinki.

\section{Medical treatment}

The routine choices of anticoagulation are VKA, LMWH, and DOACs for medical treatment of DVT in our clinic. The LMWH treatment was preferred in the patients with a history of malignancy and high risk for bleeding. In the patients with poor compliance to control visits and bloodwork including the international normalized ratio (INR) control, DOACs were preferred. The patients on warfarin treatment were called to the INR check, until they reached the therapeutic value. The control INR value was increased to 2 to 2.5 , and the followup intervals were extended (two weeks) with monthly INR monitoring.

In most patients, DVT treatment was terminated after three to six months, unless there was a risk for recurrence and a control venous Doppler ultrasonography (US) was performed. Extended treatment (>12 months) was performed in the patient group with high risk factors with recurrence and $\mathrm{PE}$. Twenty-four of Group I patients (68.6\%) and 83 of Group M (50\%) patients received extended treatment $(\mathrm{p}=0.043)$.

\section{Interventional treatment}

Interventional treatment was applied to the selected iliofemoral DVT patients who were diagnosed in the acute period. Interventional methods were preferred primarily in the patients who did not have any contraindications for thrombolytics, no restrictions for ambulation, young, and had a significant increase in diameter and tension affected the extremity.

We used CDT, ultrasound-accelerated thrombolytic therapy, and rheolytic thrombectomy (RT). All the interventional treatment methods we performed in our clinic were performed under scopy. All patients were given prone position, and they were monitored by electrocardiography, blood pressure (with cuff), and oxygen saturation $\left(\mathrm{SpO}_{2}\right)$. In the prone position, both popliteal regions of the patient were cleaned with an antiseptic solution and covered sterile. Under local anesthesia, a $6 \mathrm{~F}$ or $8 \mathrm{~F}$ sheath was placed to the ipsilateral popliteal vein under the guidance of US. A total of 5,000 IU unfractionated heparin was 
administered to all patients at the beginning of the procedure. In case of stenting, the sheath was replaced with larger sizes (10F).

Catheter-directed thrombolysis therapy was given via a multi-side hole catheter in the thrombotic segment thereby providing a thrombolytic agent (AlteplaseActilyse $50 \mathrm{mg} / 50 \mathrm{~mL}$ vial, Boehringer Ingelheim Pharma GmbH \& Co. KG, Ingelheim, Germany) locally into the thrombus. Alteplase infusion was administered at a dose of $0.05 \mathrm{mg} / \mathrm{kg} / \mathrm{h}$. Fibrinogen levels were monitored at four-h intervals during the infusion. The treatment was continued at the same dose, when the fibrinogen level was $>150 \mathrm{mg} / \mathrm{dL}$. If fibrinogen measurements fell between the range of 100 to $150 \mathrm{mg} / \mathrm{dL}$, alteplase was reduced to half-dose. When the fibrinogen was below $100 \mathrm{mg} / \mathrm{dL}$, the infusion was discontinued and infusion therapy was terminated, if the following two measurements were below $100 \mathrm{mg} / \mathrm{dL}$.

Ultrasound-accelerated CDT (EKOS Corporation Bothell, WA, USA) includes a drug application lumen, temperature sensor, and cooling system. Under the scopy, 2.2-MHz energy was applied from this catheter inserted into the lumen through the ipsilateral popliteal vein. The effect of fibrinolytic agent on thrombus was increased by providing prolongation and relaxation of the fibrin fibers through radial ultrasonic waves. The results of our experience with this method have been published previously. ${ }^{[4]}$

Rheolytic thrombectomy, power pulse spray, and rapid lyse (AngioJet Boston Scientific, Ballybrit Business Park. Galway, Ireland) aims to manage the mechanical and medical thrombectomy therapies simultaneously. The catheter has a combination of a serum-spraying mechanism and a local vacuum providing mechanism of about $600 \mathrm{mmHg}$ at a rate of about $160 \mathrm{~m} / \mathrm{s}$. In this way, the thrombus, which is mechanically fragmented by the serum jet, is removed by simultaneous aspiration. During the procedure, the effectiveness of the process is increased with the use of a local thrombolytic agent. In the patients with power pulse, $10 \mathrm{mg}$ of alteplase was placed in $500 \mathrm{~mL}$ serum and applied through thrombus and waited almost $30 \mathrm{~min}$. In the patients with a high thrombus burden, the patient was treated with alteplase $(10 \mathrm{mg} / 500 \mathrm{~mL})$ in the serum who would undergo RT (rapid lyse). In the case of fully occluded vessels, the recommendation of the manufacturer is based on a safe processing time of $600 \mathrm{sec}$. The patients with a good hydration and thrombus burden were treated for longer period (up to $770 \mathrm{sec}$ ). The patients who had a high burden of thrombus due to this restriction were treated with CDT for $24 \mathrm{~h}$ before RT. Using this method, which is a routine interventional treatment modality in our clinic, the need for thrombolytic infusion after treatment was reduced. If CDT was not used, the patients could be transferred to the ward or discharged after being monitored in the intensive care unit (ICU) on the day of the procedure. To prevent hemolysis-induced renal dysfunction, the patients were significantly hydrated and renal markers were followed during the hospital stay.

The patients who were scheduled for treatment were admitted to the ICU, and hydration was commenced along with antiplatelet and anticoagulant therapies. Heparin was used primarily during the hospital stay. The target activated partial thromboplastin time value was between 40 and $60 \mathrm{sec}$. Leg elevation was applied to all patients before and after the procedure. The duration of ICU follow-up was at least one day. The patients who underwent CDT for the treatment were kept in the ICU during infusion. In case of massive hematuria or decreased hematocrit during infusion, thrombolytic therapy was discontinued and necessary supportive therapies including transfusion were employed.

All patients were prescribed by appropriate anticoagulant and antiplatelet treatments at the time of discharge. Leg elevation and regular ambulation were also recommended to all patients.

\section{Follow-up}

The patients who were discharged were called to the first- and third-month follow-up visits and, then, to the six-month follow-up visit. All patients were informed about recommended lifestyle changes. Compression stockings were recommended to all patients and venotonic drugs were prescribed in case of significant symptoms.

\section{Statistical analysis}

Statistical analysis was performed using the SPSS for Windows version 15.0 software (SPSS Inc., Chicago, IL, USA). Descriptive data were expressed in mean \pm standard deviation (SD), median (min-max), or number and frequency. The Pearson chi-square test (or Fisher's exact test, where applicable) was used to compare discrete variables, while the independent t-test was used for continuous variables between the groups. Follow-up periods without recurrence and PE were calculated using the Kaplan-Meier method and curves were compared using a log-rank test. Recurrence during follow-up, development of 
Table 1. Risk factors for deep vein thrombosis

\begin{tabular}{lcclc}
\hline \multicolumn{1}{c}{ Group I (n=11) } & & & \multicolumn{2}{c}{ Group M (n=95) } \\
\cline { 1 - 1 } Long travel history & $\mathrm{n}$ & & $\mathrm{n}$ \\
History of orthopedic surgery & 4 & & Oncology & 24 \\
Prolonged immobilization & 3 & & Orthopedic operation/fracture & 16 \\
Pregnancy & 2 & & Prolonged immobilization & 15 \\
Use of oral contraceptives & 2 & Pregnancy & 10 \\
& 1 & Major surgery & 8 \\
& & Trauma & 8 \\
& & Coagulopathy & 8 \\
& & Long journey & 2 \\
& & Use of oral contraceptives & 1 \\
& & Other & 13 \\
\hline
\end{tabular}

PTS, and Villalta and VCSS scores were analyzed during follow-up. A $p$ value of $<0.05$ was considered statistically significant.

\section{RESULTS}

Of 217 patients, 107 (49.3\%) had complete follow-up data. The remaining of the patients were unable to be contacted by any means. A total of 29 patients in Group I (82.9\%) and 78 patients (42.9\%) in Group $M$ patients were under follow-up at the cardiovascular surgery outpatient clinic. The mean follow-up for all patients was $2.6 \pm 3.5$ years (range, 1 month to 19.7 years) with a total of 278.5 per patient-year.
The distribution of patients with risk factors is shown in Table 1. According to the developmental mechanisms, provoked DVT was observed in 11 patients (31.4\%) in Group I and in 95 patients (52.2\%) in Group $\mathrm{M}(\mathrm{p}=0.023)$. According to the lateralization, in Group I, 77.1\% $(\mathrm{n}=27)$ had left, $20.0 \%(n=7)$ had right, and 2.9\% ( $n=1)$ had bilateral DVT. The lateralization rates of Group $M$ were as follows: $58.8 \%(\mathrm{n}=107)$ left, $39.0 \%(\mathrm{n}=71)$ right, and $2.2 \%(n=4)$ bilateral DVT. Although the left leg involvement rate was higher in Group I, the difference was not statistically significant $(p=0.083)$.

Demographic data of the patients are summarized in Table 2. As expected, more patients in Group I had proximal DVT. Another striking feature is the higher percentage of provoked DVT in Group $M$ which is statistically significant.

The procedures applied to the patients are shown in Table 3. Thirty-one patients (88.6\%) who underwent interventional procedures were treated in the acute period (within the first 14 days); three (8.6\%) in the subacute period (between 14 and 28 days), and one (2.9\%) in the chronic period ( $>28$ days). The mean length of hospital stay in the ICU was $2.8 \pm 1.8$ (range, 1 to 7 ) days, and the mean duration of hospitalization was $7.6 \pm 5.1$ (range, 2 to 28 ) days. No mortality was observed in Group I perioperatively and during the first planned anticoagulation treatment.

The follow-up data of the patients are summarized in Table 4. There was no statistically significant

\begin{tabular}{|c|c|c|c|c|c|c|c|}
\hline & \multicolumn{3}{|c|}{ Group I (n=35) } & \multicolumn{3}{|c|}{ Group M (n=182) } & \multirow[b]{2}{*}{$p$} \\
\hline & $\mathrm{n}$ & $\%$ & $\mathrm{Mean} \pm \mathrm{SD}$ & $\mathrm{n}$ & $\%$ & $\mathrm{Mean} \pm \mathrm{SD}$ & \\
\hline Age (year) & & & $49.8 \pm 16.7$ & & & $51.4 \pm 13.4$ & 0.628 \\
\hline $\begin{array}{l}\text { Gender } \\
\text { Male } \\
\text { Female }\end{array}$ & $\begin{array}{l}19 \\
16\end{array}$ & & & $\begin{array}{c}100 \\
82\end{array}$ & & & 0.943 \\
\hline $\begin{array}{l}\text { LET classification } \\
\text { Class } 1 \\
\text { Class } 2 \\
\text { Class } 3 \\
\text { Class } 4\end{array}$ & $\begin{array}{c}0 \\
4 \\
28 \\
3\end{array}$ & $\begin{array}{c}0 \\
11.4 \\
80.0 \\
8.6\end{array}$ & & $\begin{array}{c}54 \\
112 \\
15 \\
1\end{array}$ & $\begin{array}{c}29.7 \\
61.5 \\
8.2 \\
0.5\end{array}$ & & 0.0001 \\
\hline $\begin{array}{l}\text { Lateralization } \\
\quad \text { Right } \\
\text { Left } \\
\text { Bilateral }\end{array}$ & $\begin{array}{c}7 \\
27 \\
1\end{array}$ & $\begin{array}{l}20.0 \\
77.1 \\
2.9\end{array}$ & & $\begin{array}{c}71 \\
107 \\
4\end{array}$ & $\begin{array}{c}39.0 \\
58.8 \\
2.2\end{array}$ & & 0.083 \\
\hline $\begin{array}{l}\text { DVT mechanism } \\
\text { Provoked } \\
\text { Non-provoked }\end{array}$ & $\begin{array}{l}11 \\
24\end{array}$ & $\begin{array}{l}31.4 \\
68.6\end{array}$ & & $\begin{array}{l}95 \\
87\end{array}$ & $\begin{array}{l}52.2 \\
47.8\end{array}$ & & 0.023 \\
\hline History of $\mathrm{PE}$ & 3 & 8.8 & & 18 & 9.9 & & 1.000 \\
\hline
\end{tabular}




\section{Table 3. Distribution of interventional treatment modalities}

\begin{tabular}{lcc} 
& $\mathrm{n}$ & $\%$ \\
\hline Rheolytic thrombectomy & 19 & 54.3 \\
Ultrasonography accelerated catheter-directed thrombolysis & 14 & 40.0 \\
Balloon angioplasty & 9 & 25.7 \\
Power pulse spray & 6 & 17.1 \\
Stent placement & 4 & 11.4 \\
Vena cava filter insertion & 2 & 5.7 \\
Catheter-directed thrombolysis & 2 & 5.7 \\
Surgical thrombectomy & 1 & 2.9 \\
\hline
\end{tabular}

difference in the mortality, recurrence, and $\mathrm{PE}$ rates during follow-up between the groups. According to the Villalta scoring at the final follow-up, the mean Villalta score was found to be $3.0 \pm 2.6$ (range, 0 to 11) in Group I and 5.4 \pm 5.2 (range, 0 to 19) in Group $M(p=0.013)$. When the number of patients with a measured Villalta score of $>5$ were compared, 32 patients (41.0\%) in Group M and one patient (3.4\%) in Group I developed PTS, indicating a statistically significant difference $(p=0.0001)$. The VCSS scores measured at the final follow-up were $2.6 \pm 3.5$ (range, 0 to 16 ) in Group I and $4.4 \pm 3.6$ (range, 0 to 18$)$ in Group M ( $\mathrm{p}=0.027)$.
The recurrence rate was $22.9 \%$ in Group I $(n=8)$ and $16.9 \%$ in Group $M \quad(n=23)$, indicating no statistically significant difference $(\mathrm{p}=0.198)$. According to the Kaplan-Meier analysis, one- and two-year recurrence-free survival rates were $91.9 \pm 3.5 \%$ and $83.1 \pm 5.8 \%$ in Group $M$ and $80.0 \pm 8.1 \%$ and $73.3 \pm 9.8 \%$ in Group I, respectively $(\mathrm{p}=0.093)$ (Figure 1). There were no patients with $\mathrm{PE}$ during and after the procedure. However, PE was seen in five patients (6.4\%) during follow-up in Group M.

Mortality was seen in two patients $(5.7 \%)$ in Group I and in six patients (13.9\%) in Group M which were not related to DVT. According to Kaplan-Meier method, one-year and two-year survival rates were $96.7 \pm 3.3 \%$ in Group I and $94.4 \pm 2.3 \%$ in Group M, indicating no statistically significant difference between the groups (Figure 2).

Anticoagulant-associated bleeding rates were 27.6\% $(\mathrm{n}=8)$ in Group I and $41.0 \%$ in Group M $(\mathrm{n}=32)$, indicating no statistically significant difference between the groups $(\mathrm{p}=0.195)$. When the anticoagulant therapies used in 107 patients with complete follow-up data, 17 patients $(15.9 \%)$ received LMWH, 32 patients $(29.9 \%)$ received warfarin, 50 patients $(46.7 \%)$ received DOACs, seven patients $(6.5 \%)$ were on aspirin, and

Table 4. Follow-up data

\begin{tabular}{|c|c|c|c|c|c|c|c|c|c|}
\hline & \multicolumn{4}{|c|}{ Group I (n=35) } & \multicolumn{4}{|c|}{ Group $\mathrm{M}(\mathrm{n}=182)$} & \multirow[b]{2}{*}{$p$} \\
\hline & $\mathrm{n}$ & $\%$ & Mean $\pm S D$ & Min-Max & $\mathrm{n}$ & $\%$ & Mean $\pm S D$ & Min-Max & \\
\hline Completed follow-up & 29 & 82.9 & & & 78 & 42.9 & & & 0.0001 \\
\hline Pulmonary embolism & 0 & 0 & & & 5 & 6.4 & & & 0.614 \\
\hline Recurrence & 8 & 22.9 & & & 23 & 13.9 & & & 0.198 \\
\hline Mortality & 2 & 5.7 & & & 6 & 3.3 & & & 0.618 \\
\hline Mean treatment time (month) & & & $32.3 \pm 25.0$ & & & & $33.9 \pm 39.6$ & & \\
\hline Prolonged treatment* & 24 & 68.6 & & & 83 & 50.0 & & & 0.043 \\
\hline \multicolumn{10}{|l|}{ Survival (year) } \\
\hline General & & & $2.1 \pm 2.1$ & $0.0-19.6$ & & & $2.4 \pm 3.5$ & $0.0-21.0$ & 0.706 \\
\hline Without recurrence & & & $1.6 \pm 1.7$ & & & & $2.6 \pm 3.7$ & & 0.052 \\
\hline Without pulmonary embolism & & & $1.7 \pm 1.7$ & & & & $2.9 \pm 3.9$ & & 0.753 \\
\hline Last Villalta score & & & $3.0 \pm 2.6$ & & & & $5.4 \pm 5.2$ & & 0.013 \\
\hline Villalta $>5$ & 1 & 3.4 & & & 32 & 41.0 & & & 0.0001 \\
\hline Last venous clinical severity score & & & $2.6 \pm 3.5$ & & & & $4.4 \pm 3.6$ & & 0.027 \\
\hline Venous clinical severity score $>8$ & 2 & 6.9 & & & 7 & 9.0 & & & 1.000 \\
\hline Anticoagulant-associated bleeding & 8 & 27.6 & & & 32 & 41.0 & & & 0.195 \\
\hline
\end{tabular}




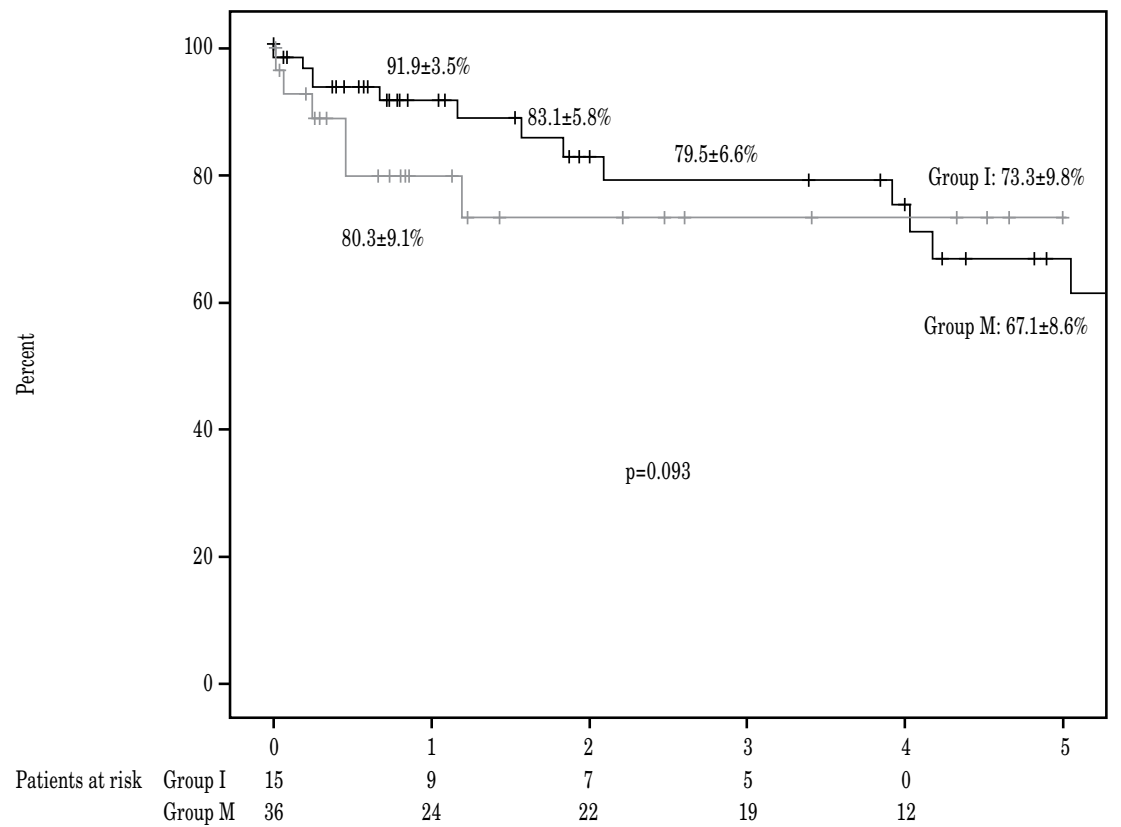

Figure 1. Recurrence-free survival curves.

one patient $(0.9 \%)$ did not receive the recommended treatment.

Two patients had massive hematuria. The first of these patients (21 years old, male) was on CDT after RT, and thrombolytic infusion was discontinued due to hematuria. The other patient (86 years old, female) received ultrasound-accelerated thrombolytic therapy. Thrombolytic infusion was discontinued due to hematuria, and two units of erythrocyte suspension and one unit of fresh frozen plasma replacement were performed. The patient was followed for seven days in the ICU and 13 days in the hospital in total, and her complaint regressed with supportive care. Direct oral anticoagulants were used for post-discharge follow-up. She had retroperitoneal hemorrhage and she died from an unknown reason 27 months after the procedure.

One patient (2.9\%) who was treated with RT without a thrombolytic agent and DOAC therapy had intraventricular bleeding two months after the procedure and died. Another patient who was

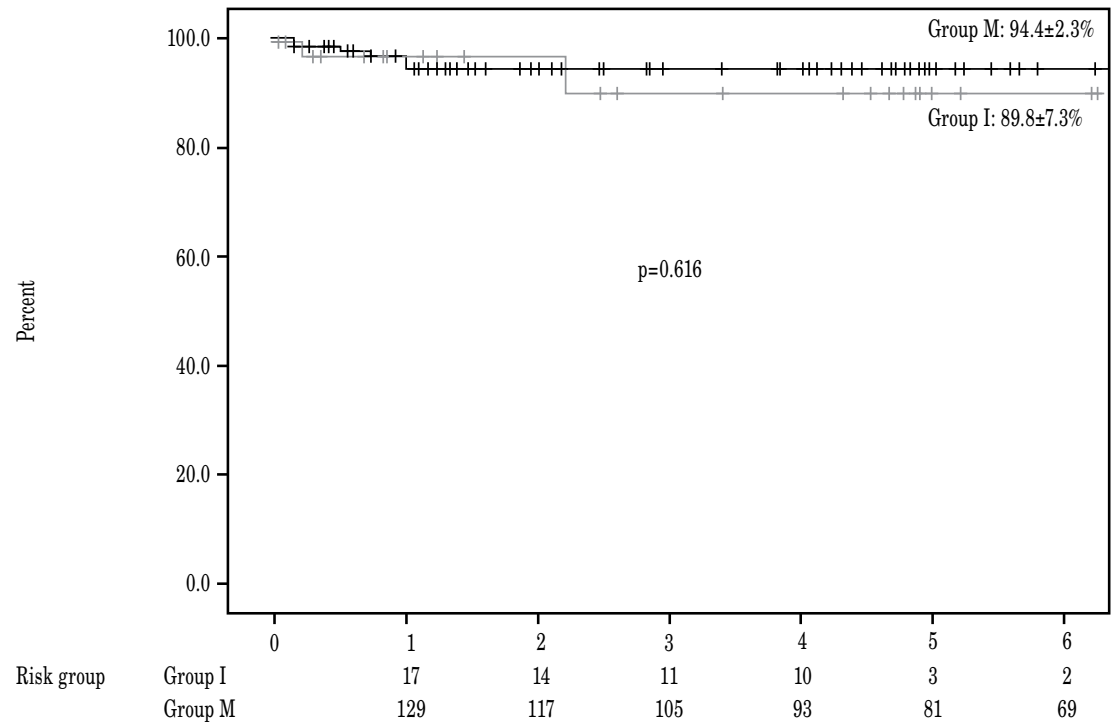

Figure 2. Kaplan-Meier survival curves. 
lost-to-follow-up after the procedure was prescribed with warfarin, and no major complication was observed, except for mild ecchymosis, as reported by the patient during telephone interview. Eighteen months after the procedure, the patient was admitted to the emergency department with a complaint of worsened general condition. However, a detailed medical data was unable to be reached, as the patient died in an external center with an unknown reason.

Access site-related hematoma occurred in one patient $(2.9 \%)$ in the popliteal region which was spontaneously regressed completely during follow-up.

In six patients (3.3\%) in Group M, major bleeding (gastrointestinal bleeding $\mathrm{n}=5$, uterus hematoma $\mathrm{n}=1$ ) and minor bleeding in seven patients (ecchymosis, gingivalbleeding, and increased menstruationbleeding) were observed. During follow-up, six patients (3.3\%), all of whom were malignant, died. Two of these patients (1.1\%) were those with major bleeding due to anticoagulant therapy (gastrointestinal bleeding).

Acute renal failure developed in one patient (2.9\%) during the ICU stay. The patient received dialysis treatment three months after discharge, and his blood urea and creatinine levels returned to normal and he remained free from dialysis treatment for five months.

\section{DISCUSSION}

In the present study, interventional treatment and medical treatment for lower extremity DVT were compared. The main findings of our study included that a higher number of patients in Group I received extended treatment and the mean PTS rate calculated by the VCSS and Villalta scores were lower in these patients in the mid-term.

The Catheter-directed Venous Thrombolysis $(\mathrm{CaVENT})$ and ATTRACT trials are two major studies in which interventional and medical treatment methods are compared in terms of PTS. The ATTRACT trial ${ }^{[3]}$ resulted in the failure of interventional treatments to show the expected benefit in preventing PTS development, compared to medical therapy (PTS rates were $48 \%$ in pharmacomechanical thrombectomy group and $47 \%$ in control group). In our study, the severity of symptoms was significantly lower as evident with significantly lower Villalta and VCSS scores in Group I (Table 4). When the ATTRACT trial was compared with our study, we believe that the difference in PTS reports was related to the underestimation of the patients' symptoms. In our clinic, interventional treatment modalities were preferred in Class 3-4 patients according to the LET classification. We found that the success of the Group I was significantly higher than Group M. In the CaVENT trial, which first demonstrated the efficacy of thrombolytic therapy, the five-year results of 176 patients ( $n=87$ CDT, $n=89$ controls) were evaluated. ${ }^{[6]}$ The PTS development rates were $43 \%$ and $71 \%(\mathrm{p}<0.0001)$ in the CDT group and in the control group, respectively.

In a study including 38 patients who underwent RT + CDT, the PTS development rate was reported as $17 \%$ during a 20 -month follow-up period. ${ }^{[7]}$ In another study, 68 patients who underwent RT with the AngioJet were evaluated retrospectively and the mean pre- and postoperative VCSS scores were found to be $13.1 \pm 2.2$ and $4.0 \pm 1.3$, respectively $(p=0.01)$, while the mean Villalta scores decreased from $12.9 \pm 2.8$ to $5.5 \pm 1.4$ postoperatively $(\mathrm{p}<0.001){ }^{[8]}$ Only three patients had minor bleeding. However, none of the patients had major complications such as major bleeding, PE, or death. Therefore, the results of our study are consistent with previous studies suggesting that PTS can be significantly reduced by the interventional treatment of DVT. The bleeding complications after CDT may be impractical; however, as previously been discussed, ${ }^{[3]}$ even after the ATTRACT trial, the practice in DVT treatment remained unchanged for the physicians. The main advantage of the interventional treatment modality is that improved Villalta scores are prominent with low morbidity rates in selected cases.

In the Early Coronary Angiography Versus Delayed Coronary Angiography (PEARL) trial, 329 patients had a recurrence rate of $94 \%, 87 \%$, and $83 \%$ at three, six, and 12 months during follow-up, respectively. ${ }^{[9]}$ Major bleeding rate was $3.6 \%$ and none was associated with the AngioJet use. In our study, the mean rate of the patients without recurrence in Group I was $73.3 \pm 9.8$ at four years; the mean survival without recurrence was $1.6 \pm 1.8$ years; and the total bleeding rate was $25.7 \%(n=9)$. The proportion of the patients receiving prolonged treatment in the interventional treatment group was significantly higher. This high rate should be considered as another factor which reduces the recurrence rate.

In a study by Shen et al., ${ }^{[10]} 79$ patients who underwent RT (with power pulse spray) and 119 patients with CDT were examined for kidney injury. Acute renal injury was $22.8 \%$ in patients with RT+thrombolytic therapy $(p=0.013)$. In our study, one 
patient who developed acute renal failure had dialysis treatment for three months and, then, renal functions returned to normal. Renal injury after RT is an important risk for surgeons to consider. The low rate of renal dysfunction in our study group can be explained by the liberal hydration protocol applied to the patients in our clinic.

In their study, Sharifi et al. ${ }^{[11]}$ published the results of 133 patients treated with iliac and femoropopliteal venous stents. The reported stent thrombosis rate was $4 \%$. There is also a study regarding the risk for intimal hyperplasia and DVT in the contralateral extremity after migration or extension of the stent to the inferior vena cava. ${ }^{[12]}$ The stent patency rates of the patients with stent implantation by direct and progressive methods were found to be $93.5 \%$ and $97.8 \%$, respectively $(\mathrm{p}=0.323)$ and the mean Villalta scores were found to be $4.2 \pm 2.5$ and $2.1 \pm 1.9$, respectively. ${ }^{[13]}$ In addition, one-year patency rates were reported as higher than $90 \%$ with specialized vein stents. However, the patency rates for Wallstent ${ }^{\circledR}$ were previously reported as below $70 \%$, particularly in PTS patients. ${ }^{[14]}$

In the present study, we applied extended treatment ( $>12$ months) to the patient group with high risk factors with recurrence and PE. In 24 patients $(68.6 \%)$ of Group I and 83 patients (50\%) of Group M received extended treatment $(p=0.043)$. We believe that the prolonged treatment of most of the Group I patients has a share in preventing the development of PTS in this patient group.

\section{Limitations of the study}

The main limitations of the study are its retrospective nature and missing patient data. Patients' communication data were incomplete or inaccurate, patients did not attend to the follow-up regularly or follow-up visits in external centers. Therefore, the efficacy of the treatments which were applied was unable to be evaluated individually. Another limitation is the probability of incorrect entry of records in the hospital registry systems. We believe that many patients were unable to be drawn from the hospital records due to miscoding or other problems with the electronic record systems. The patients with missing data (incomplete follow-up data could not be completed) were excluded from the analysis in terms of recurrence and development of PTS. However, the rate of PTS development in Group $M$ was found to be close to the ATTRACT trial. In Group I, low rates were found. Although we acknowledge that these low rates are due to lack of data, it should be also considered that there was a significant difference between the patients.

In conclusion, the PTS development rate decreased significantly over two years after interventional treatments. Symptoms were significantly lower as measured with the Villalta and VCSS scores. Based on these findings, necessary infrastructure should be developed to increase interventional treatment practices, and physicians should be able to apply these methods effectively with an increased learning curve through relevant training programs. Thus, a considerable progress can be made in terms of DVT treatment and prevention of complications.

\section{Declaration of conflicting interests}

The authors declared no conflicts of interest with respect to the authorship and/or publication of this article.

\section{Funding}

The authors received no financial support for the research and/or authorship of this article.

\section{REFERENCES}

1. Vázquez FJ, Posadas-Martínez ML, Vicens J, González Bernaldo de Quirós F, Giunta DH. Incidence rate of symptomatic venous thromboembolic disease in patients from a medical care program in Buenos Aires, Argentina: a prospective cohort. Thromb J 2013;11:16.

2. Kearon C, Akl EA, Ornelas J, Blaivas A, Jimenez D, Bounameaux $\mathrm{H}$, et al. Antithrombotic therapy for VTE Disease: CHEST guideline and expert panel report. Chest 2016;149:315-52.

3. Marston WA. Results of the ATTRACT trial do not change the management of acute deep vein thrombosis. J Vasc Surg Venous Lymphat Disord 2018;6:5-6.

4. Polat A, Ketenciler S, Boyacıŏlu K, Yücel C, Yıldızhan İ, Kük ZG, et al. Accelerated catheter-directed thrombolytic treatment in deep venous thrombosis: mid-term results. Turk Gogus Kalp Dama 2015;23:485-92.

5. Arnoldussen $\mathrm{CW}$, Wittens $\mathrm{CH}$. An imaging approach to deep vein thrombosis and the lower extremity thrombosis classification. Phlebology 2012;27:143-8.

6. Haig Y, Enden T, Slagsvold CE, Sandvik L, Sandset PM, Kløw NE. Residual rates of reflux and obstruction and their correlation to post-thrombotic syndrome in a randomized study on catheter-directed thrombolysis for deep vein thrombosis. J Vasc Surg Venous Lymphat Disord 2014;2:123-30.

7. Liu G, Cai Y, Philippi T, Kalla P, Scheidt D, Richards $\mathrm{J}$, et al. Distribution of total and methylmercury in different ecosystem compartments in the Everglades: implications for mercury bioaccumulation. Environ Pollut 2008;153:257-65.

8. Dumantepe M, Uyar I. The effect of Angiojet rheolytic thrombectomy in the endovascular treatment of 
lower extremity deep venous thrombosis. Phlebology 2018;33:388-96.

9. Garcia MJ, Lookstein R, Malhotra R, Amin A, Blitz LR, Leung DA, et al. Endovascular Management of Deep Vein Thrombosis with Rheolytic Thrombectomy: Final Report of the Prospective Multicenter PEARL (Peripheral Use of AngioJet Rheolytic Thrombectomy with a Variety of Catheter Lengths) Registry. J Vasc Interv Radiol 2015;26:777-85.

10. Shen Y, Wang X, Jin SS, Zhang RL, Zhao WJ, Chen G. Increased risk of acute kidney injury with percutaneous mechanical thrombectomy using AngioJet compared with catheter-directed thrombolysis. J Vasc Surg Venous Lymphat Disord 2019;7:29-37.

11. Sharifi M, Javadpoor SA, Bay C, Mehdipour M, Emrani F, Sharifi J. Outcome of stenting in the lower extremity venous circulation for the treatment of deep venous trrombosis. Vasc Dis Manag 2010;7:233-9.

12. Le TB, Lee TK, Park KM, Jeon YS, Hong KC, Cho SG. Contralateral deep vein thrombosis after iliac vein stent placement in patients with May-Thurner syndrome. J Vasc Interv Radiol 2018;29:774-80.

13. Liu G, Qin J, Cui C, Ye K, Shi H, Liu X, et al. Comparison of direct iliofemoral stenting following angiojet rheolytic thrombectomy vs staged stenting after angiojet rheolytic thrombectomy plus catheter-directed thrombolysis in patients with acute deep vein thrombosis. J Endovasc Ther 2018;25:133-9.

14. Shamimi-Noori SM, Clark TWI. Venous stents: Current status and future directions. Tech Vasc Interv Radiol 2018;21:113-6. 